\title{
Wind observations of low energy particles within a solar wind reconnection region
}

\author{
K. E. J. Huttunen ${ }^{1,2}$, S. D. Bale ${ }^{1,3}$, and C. Salem ${ }^{1}$ \\ ${ }^{1}$ Space Sciences Laboratory, University of California, 7 Gauss Way, Berkeley, CA 94720, USA \\ ${ }^{2}$ Department of Physics, P.O. Box 64, 00014, Univeristy of Helsinki, Finland \\ ${ }^{3}$ Department of Physics, University of California, Berkeley, CA 94720, USA
}

Received: 11 June 2007 - Revised: 24 June 2008 - Accepted: 30 June 2008 - Published: 12 September 2008

\begin{abstract}
We report characteristics of thermal particle observations during the encounter of the Wind satellite with the separatrix and the outflow domains of a reconnection event on 22 July 1999 in the solar wind. During the studied event the electrostatic analyzers on Wind were transmitting threedimensional electron and proton distributions in a burst mode every $3 \mathrm{~s}$, the spin period of the spacecraft. The event was associated with a magnetic shear angle of $114^{\circ}$ and a large guide magnetic field. The observations suggest that Wind crossed the separatrix and outflow regions about a thousand of ion skin depths from the X-line. At the leading separator boundary, a strong proton beam was identified that originated from the direction of the X-line. In the separatrix and the outflow regions, the phase space distributions of thermal electrons displayed field aligned bidirectional anisotropy. During the crossings of the current sheets bounding the outflow region, we identified two adjacent layers in which the dominant thermal electron flows were towards the $\mathrm{X}$-line at the inner edges of the current sheets and away from the X-line at the outer edges. Interestingly, simulation studies and observations in the Earth's magnetosphere have revealed that the electron flows are reversed, consistent with the Hall current system.
\end{abstract}

Keywords. Interplanetary physics (Solar wind plasma) Space plasma physics (Charged particle motion and acceleration; Magnetic reconnection)

\section{Introduction}

Magnetic reconnection is a fundamental process in space plasma that enables fast release of magnetic energy and changes topology of the magnetic field. In the past, in-situ observations of magnetic reconnection have been made al-

Correspondence to: K. E. J. Huttunen

(huttunen@ssl.berkeley.edu) most solely in the Earth's magnetosphere where reconnection is typically random and highly dynamic. Recent studies have established the solar wind as an important new laboratory to investigate in-situ the microphysical processes related to reconnection (Gosling et al., 2005a; Phan et al., 2006; Huttunen et al., 2007). In the solar wind, the boundary conditions on the opposite sides of the reconnection current sheets are relatively stable and the reconnection process appears to be largely undriven and quasi-steady (Phan et al., 2006). Furthermore, the solar wind quickly carries the reconnection sites past the observing spacecraft making the separation between spatial and temporal variations easier.

Reconnection events in the solar wind are typically identified in the form of "Petschek-type" exhausts where the plasma outflow from the X-line is bounded by Alfvén waves (Gosling et al., 2005a, 2006a). Figure 1 illustrates the configuration of the reconnection region following the scenario of fast magnetic reconnection by Petschek (1964). The dashed box outlines the X-line region (e.g. Mozer, 2005) where reconnection is initiated and that is only a few ion skin depths $\left(\lambda_{i}=c / \omega_{p i}\right.$, where $\omega_{p i}$ is the ion plasma frequency) in size. As reconnection proceeds the initial current sheet bifurcates into two pairs of Alfvén waves at which the magnetic field changes direction and the main energy conversion takes place. Separatrices that are shown by the pair of black lines in Fig. 1 represent the most recently reconnected field lines and mark the outer boundaries of the reconnection region.

The extended green-hatched area in Fig. 1 between the separatrices and the plasma outflow region is called the "separatrix region" (e.g. Vaivads et al., 2006). In-situ observations during reconnection events at the dayside magnetopause have revealed highly structured and dynamic electric fields in separatrix regions and shown that the separatrix region can maintain its internal structure over at least several hundreds of ion skin depths (André et al., 2004; Retinó et al., 2006; Khotyaintsev et al., 2006; Wygant et al., 2005). Wygant et al. (2005) also demonstrated that the thickness of

Published by Copernicus Publications on behalf of the European Geosciences Union. 


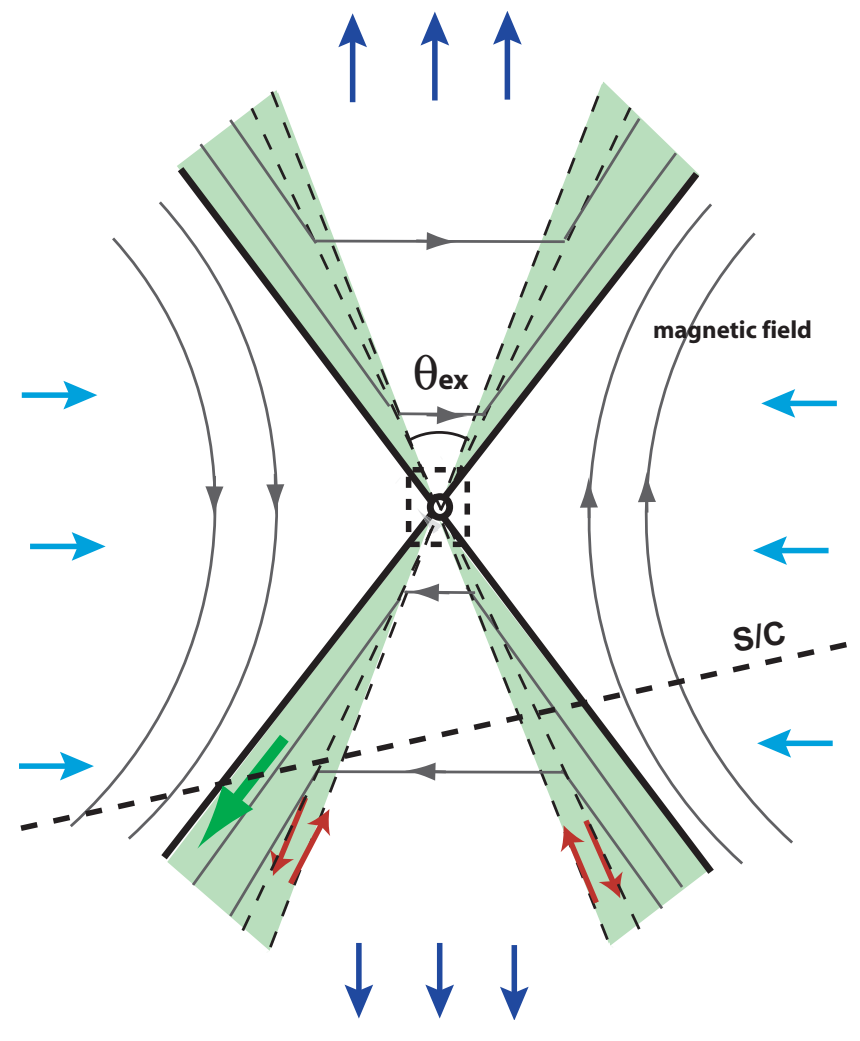

Fig. 1. Illustration of a reconnection region in the plane of reconnection. Thick black lines show the separatrices and the dashed box outlines the $\mathrm{X}$-line region. The green-hatched area depicts the separatrix region where the dashed lines bound the current sheets. Light (dark) blue arrows denote the plasma inflow (outflow). Green arrow shows the direction of the proton beam and the red arrows show the dominant directions of the electron flow during the current sheet crossings.

the current sheets that bound the outflow region (marked by two pairs of dashed lines in Fig. 1) increases with an increasing distance from the $\mathrm{X}$-line. In addition, various plasma wave modes have been reported within the separatrix regions in the Earth's magnetosphere (Bale et al., 2002; Matsumoto et al., 2003; Cattell et al., 2005) and also in the solar wind (Huttunen et al., 2007).

In this paper, we investigate for the first time high-time resolution particle measurements associated with the separatrix and the outflow domains of a reconnection region in the solar wind. The reconnection event studied here took place on 22 July 1999 and its passage by the Wind satellite lasted $54 \mathrm{~s}$ (time between the observation of the separatrices). We use observations from the Electron and Proton electrostatic analyzers (EESA-L and PESA-L respectively) included in the 3-D Plasma and Energetic Particles (3-DP) experiment (Lin et al., 1995) on-board the Wind satellite. EESA-L and PESA$\mathrm{L}$ were operating in a burst mode transmitting 3-s threedimensional particle distributions; these high time resolution measurements enabled this study. In Sect. 2, we describe the data sets used. An overview of solar wind plasma and magnetic field observations is presented in Sect. 3.1 and particle distributions are examined in Sect. 3.2. Section 4 provides a discussion of the observations and summarizes the results.

\section{Data sets}

The Wind satellite was launched 1 November 1994 with the goal to monitor plasma processes in the near-Earth solar wind and in the Earth's magnetosphere. During the event studied here, Wind was located in the solar wind $182 R_{E}$ upstream of the Earth close to the Sun-Earth line.

We primarily use observations obtained with the EESA-L and PESA-L electrostatic analyzers of the Wind 3-DP experiment. EESA-L provides full three-dimensional distributions of electrons from a few eV to $1.1 \mathrm{keV}$ in 15 logarithmically spaced energy steps and full $4 \pi$ coverage in one spacecraft spin $(\sim 3 \mathrm{~s})$. The PESA-L analyzer measures the solar wind ions from $3 \mathrm{eV}$ to $30 \mathrm{keV}$ at the same $3 \mathrm{~s}$ resolution. PESA-L was specifically designed to sample the solar wind protons by tracking the core of their distribution. EESA-L and PESA-L can be put into a burst mode, returning contiguous spin resolution 3-D distribution functions. In normal mode of operation in the solar wind, 3-D proton and electron distributions are available only every $48 \mathrm{~s}$ and $96 \mathrm{~s}$, respectively; however, moments of both proton and electron distributions (density, velocity, and temperature) are calculated on board and available at a $3 \mathrm{~s}$ resolution. The on-board proton moment calculation relies on an algorithm that separates the alpha particles from the main proton population. So, as a point of comparison in our study, we also compute the moments separately by integrating the measured ion distribution from PESA-L.

EESA-L and PESA-L measurements are converted into units of distribution functions and transformed into the solar wind rest frame using the on-board solar wind proton velocity. The electron measurements are corrected for the spacecraft potential. The spacecraft potential was estimated using the electron density obtained from the Thermal Noise Receiver (TNR) of the WAVES experiment on Wind by the method addressed by Salem et al. (2001). The TNR electron density is determined every $4.5 \mathrm{~s}$ from the spectrum of the quasi-thermal noise around the electron plasma frequency and it is quite immune to the spacecraft charging effects.

Magnetic field measurements are obtained with a hightime resolution ( $\sim 11$ vectors per second) from the Magnetic Field Investigation (MFI) experiment. A signal in the magnetic field data due to the spacecraft spin at $0.33 \mathrm{~Hz}$ have been carefully removed using a finite impulse response (FIR) filter. In the following, the magnetic field and the velocity data will be shown in the boundary normal coordinate system $L M N$ obtained from a minimum variance analysis (Sonnerup and Cahill, 1967) of the magnetic field vector across the entire exhaust, $L$ being the direction along the 

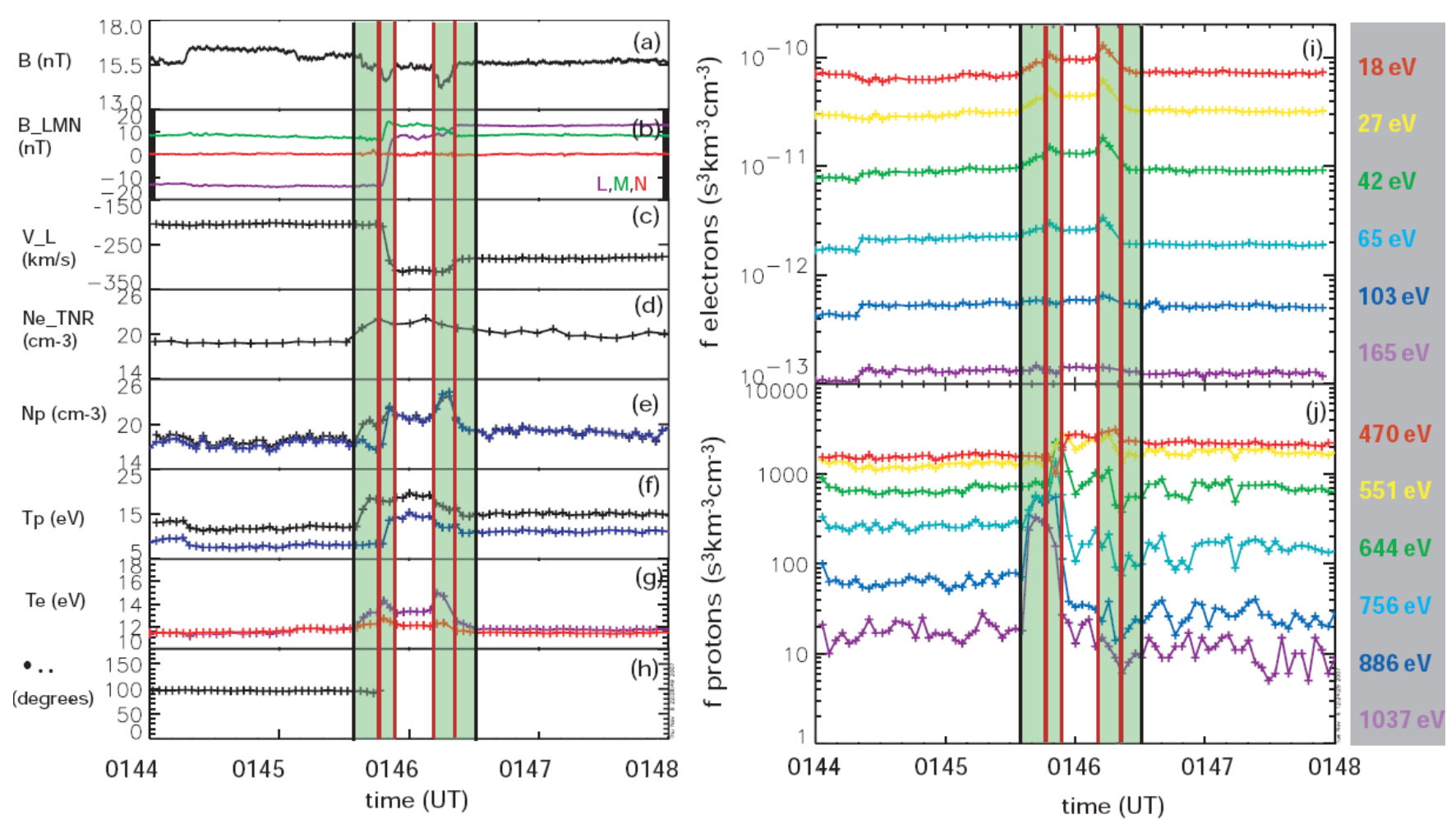

Fig. 2. Magnetic field and solar wind plasma measurements by the Wind satellite during a reconnection event on 22 July 1999 . The panels on the left show: (a) the magnetic field strength, (b) the magnetic field components in the $L M N$-coordinate system, (c) the solar wind velocity in the $L$-direction, (d) the electron density from the TNR instrument, (e) the proton density, calculated on-board (blue line) and integrated from the 3-D distribution functions (black line), (f) the proton temperature, calculated on-board (blue line) and integrated from the 3-D distribution functions (black line), (g) the electron temperature parallel (purple line) and perpendicular (red line) to the magnetic field, and (h) the angle $\theta_{B N}$ between the magnetic field and the model bow shock normal. The panels on the right show: (i) the electron phase space densities, transformed into the solar wind rest frame and integrated over all pitch angles, from 18 to $165 \mathrm{eV}$, and (j) the proton phase space densities from 470 to $1037 \mathrm{eV}$, also transformed into the solar wind rest frame and integrated over all pitch angles. The estimated locations of the separatrices are indicated by the black vertical lines, and the current sheet crossings are delimited by the two pair of red lines. The outflow region is confined between two innermost red lines.

anti-parallel magnetic field direction, $M$ the direction along the $\mathrm{X}$-line, and $N$ the direction along the overall exhaust normal.

\section{Observations}

\subsection{Overview of the reconnection event}

Figure 2 displays the magnetic field and solar wind plasma measurements from the Wind satellite during a 4-min period spanning the reconnection region on 22 July 1999. The observation of the plasma outflow along the $L$-direction confined between two regions of magnetic field directional changes suggest that reconnection was on-going at the time the event passed the Wind satellite. Multisatellite observations have indicated that near $1 \mathrm{AU}$ solar wind reconnection can be quasi-stationary on timescales at least up to couple of hours (Phan et al., 2006). The total magnetic field rota- tion across the outflow and separatrix regions was $114^{\circ}$ and there was a significant guide field $\left(B_{M}\right)$ associated with this event (the average ratio of $B_{M}$ to the total magnetic field was 0.85 ). A more detailed discussion on the magnetic field and plasma observations associated with this event is provided by Huttunen et al. (2007).

The crossing distance of the Wind satellite from the $\mathrm{X}$-line can be estimated by assuming a configuration as in Fig. 1 in which the width of the outflow region increases linearly with the distance away from the X-line. The opening angle of the exhaust, $\theta_{e x}$ (Fig. 1) can be approximated by the dimensionless reconnection rate $M_{A i}=\Delta V_{N} / 2 V_{A i}$ (see Huttunen et al., 2007 , for details). Using a shift of $6 \mathrm{~km} / \mathrm{s}$ in $V_{N}$ across the exhaust and an Alfven speed $V_{A i}$ of $70 \mathrm{~km} / \mathrm{s}$ in the inflow region yields a reconnection rate of 0.04 . This value is close to the reconnection rate 0.03 estimated previously for reconnection events in the solar wind (Phan et al., 2006; Davis et al., 2006) and is in the range of values for fast magnetic reconnection. 
This estimated reconnection rate yields the average spacecraft crossing distance of $4.4 \times 10^{4} \mathrm{~km}\left(\sim 7\right.$ Earth radii, $\left.R_{E}\right)$ from the $\mathrm{X}$-line or about a thousand ion skin depths using the average ion skin depth $\lambda_{i}$ of $50 \mathrm{~km}$ in the reconnection region. Note that any slight uncertainties in the exhaust normal determination would result in significant error in $V_{N}$ and thus the obtained reconnection rate and the crossing distance are only estimates.

The two pairs of red solid lines in Fig. 2 mark the current sheet crossings based on the start and end times of the dips in the magnetic field magnitude and the magnetic field directional changes. The current sheet within the front separatrix region was observed between 01:45:46 and 01:45:54 UT and in the trailing separatrix region between 01:46:11 and 01:46:21 UT, corresponding to widths of $19 \lambda_{i}$ and $24 \lambda_{i}$, respectively (using the average normal velocity of $\sim 120 \mathrm{~km} / \mathrm{s}$ ).

According to the Petcheck model of fast magnetic reconnection, the opening angle of the separatrices can have values up to twice the opening angle of the exhaust. Using the value $2 \times \theta_{e x}$ for the opening angle of the sepratrices places the separatrices at 01:45:36 UT and at 01:46:30 UT, which are shown by the black solid lines in Fig. 2 .

Figure 2 shows that the outflow region is characterized by increased particle temperatures and densities. As will be discussed in Sect. 3.2, a strong proton beam is observed at the leading separator boundary. The onboard moment algorithm truncates the moment integrals in energy to estimate separate proton and alpha distributions. The proton beam then appears at "alpha" energies and this results in a poor estimate of density and temperature (in the on board moments). This can be seen in Fig. 2e, which shows both the on-board proton density (blue line) and the density computed from the 3-D distribution (black line) during the 4-min interval considered here. The contribution of the alpha particles to the ion density is usually small, so the difference between the densities in Fig. 2e represents the effect of the proton beam. Similarly, Fig. $2 \mathrm{f}$ shows the difference between the on-board proton temperature (blue line) and the integrated one (black line); the offset between the two temperatures is due to the alpha particles, but one can clearly see the rise in the ion temperature, at the same time as the rise in ion or electron density (Fig. 2d), due to the presence of the proton beam. At the trailing separator edge, the transition from outside to inside the outflow region is slow-mode like characterized by the increases in the proton density and temperature and the decrease in the magnetic field magnitude.

Figure $2 \mathrm{~g}$ shows an increase of the electron temperature during the encounter with the reconnection region. The electron temperature increases mainly in the direction parallel to the magnetic field (purple line) and exhibits distinct maxima when the current sheets were crossed. A lack of Increase in the electron temperature has been reported in the previous studies of solar wind reconnection events (e.g. Gosling et al., 2005a, 2007).
Figure $2 \mathrm{~h}$ gives the angle between the magnetic field vector and the modeled bow shock normal. We have used a pressure scaled bow shock model (Slavin and Holzer, 1981) and assumed straight magnetic field lines. No points are plotted when the magnetic field lines do not intersect with the bow shock. According to the model, Wind was magnetically connected to the bow shock from 22 July 1999 at 00:55 UT until the front edge of the reconnection outflow region. The possible contamination of the particle observations by the backscattered electrons from the bow shock will be discussed in Sect. 3.2.

\subsection{Particle observations}

The electron and proton phase space densities for six different energy channels of EESA-L and PESA-L, integrated for all pitch angles, are presented in Fig. $2 \mathrm{i}$ and $\mathrm{j}$. Electron phase space densities (Fig. 2i) exhibit a distinct maximum at energies up to $65 \mathrm{eV}$ during Wind encounter with the current sheets. As shown in Fig. 2g, these sharp increases are mainly due to the increased electron temperature parallel to the magnetic field.

The most remarkable feature in the proton phase space densities in Fig. $2 \mathrm{j}$ is a strong enhancement at energies of $644 \mathrm{eV}$ and above within the front separatrix region. The lower energies (470 and $551 \mathrm{eV}$ ) exhibit an overall enhancement in the phase space densities when Wind enters the outflow region. The enhancement at energies above $644 \mathrm{eV}$ corresponds to the presence of a proton beam. This can be seen on Fig. 3, which displays three proton distribution functions, just outside the reconnection region at 01:45:29 UT (left), within the leading separatrix region (middle) and in the outflow region (right) at 01:45:57 UT. These distributions (acquired in $3 \mathrm{~s}$ ) are represented in the solar wind rest frame and in the local magnetic field coordinate system. Before Wind encounters the reconnection region, the distributions are quasi-isotropic. In the front separatrix region, a welldefined proton beam is observed flowing along the magnetic field thus indicating that the proton beam originates from the direction of the X-line and not from the direction of the bow shock, which is in the opposite direction (see below, analysis of Fig. 4). The fact that the beam structure changes during the transition from the front separatrix region to the outflow region, and the beam speed decreases, could be due to a timeof-flight effect (e.g. Filbert and Kellogg, 1979).

Figure 4 shows the pitch angle spectrograms for electrons over the same energy range of EESA-L as given in Fig. 2 i. The most prominent feature in the spectrograms of 165 and $103 \mathrm{eV}$ electrons is a strong enhancement at pitch angles $<90^{\circ}$ throughout the investigated 4-min interval. This feature corresponds to the well-known solar wind electron strahl that consists of field-aligned outward flowing suprathermal electrons originating from the hot solar corona. The strahl is observed uninterrupted during the Wind encounter with the reconnection region and therefore the magnetic field lines 

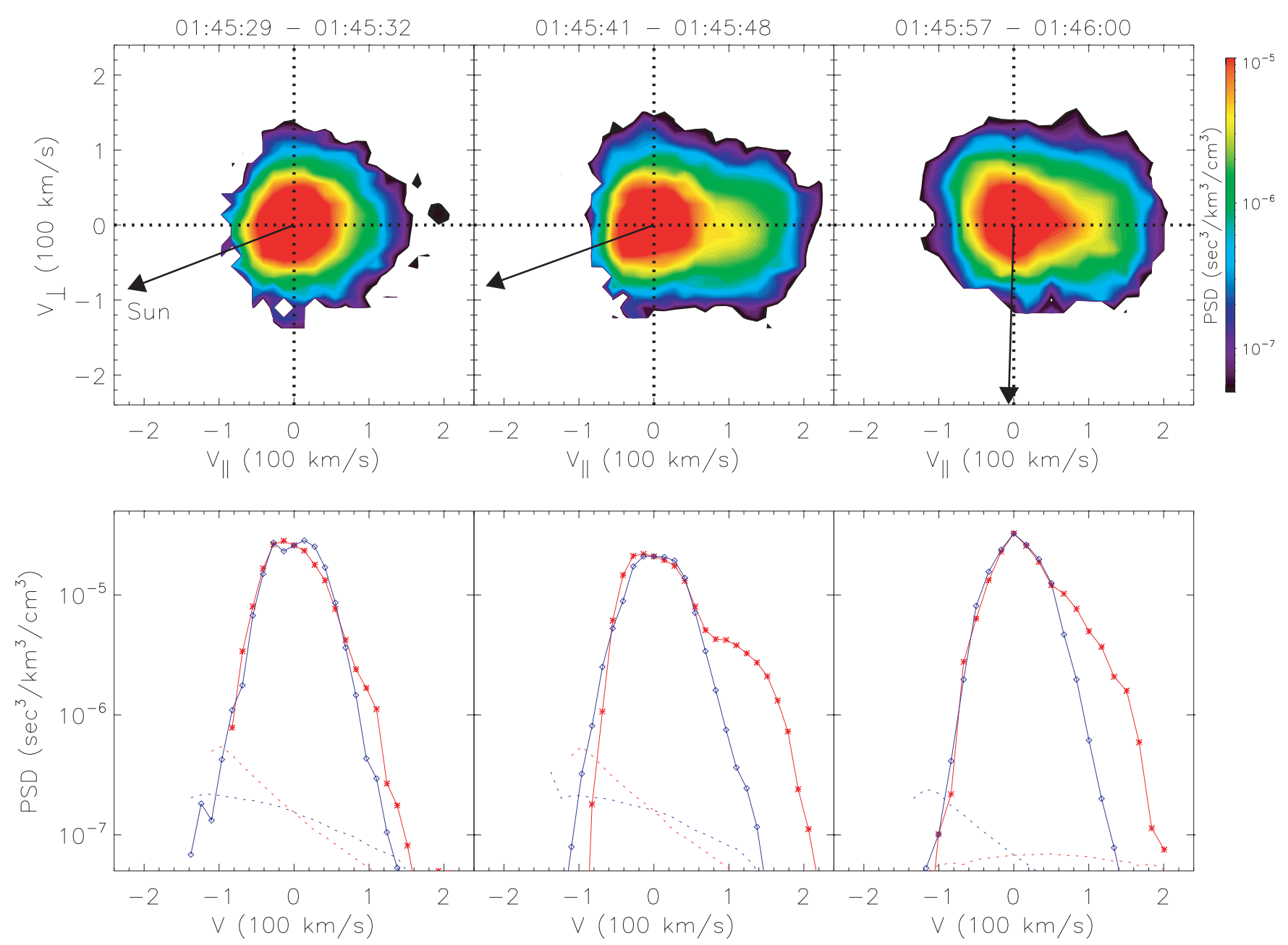

Fig. 3. Wind 3D-P PESA-L ion distribution functions (left) just outside the reconnection region at 01:45:29 UT; (middle) within the front separatrix region from 01:45:41 to 01:45:48 (average of two consecutive distributions); (right) in the outflow region at 01:45:57. Data are presented in the solar wind frame and in the magnetic field coordinate system. The top panels show the 2-D distributions in the plane [ $\left.v_{\|}, v_{\perp}\right]$ ( $v_{\|}$and $v_{\perp}$ being the velocities in the direction parallel and perpendicular to the local magnetic field). The direction to the Sun (basically the Sun-Earth direction) is indicated by an arrow. The color coding indicates the amplitude of the distribution function in units of $\mathrm{s}^{3} \mathrm{~km}^{-3} \mathrm{~cm}^{-3}$. Cuts of the distributions along the direction parallel (in red) and/or perpendicular (in blue) to the magnetic field are shown on the bottom panels. The dashed lines represent the one-count level distribution.

in the reconnection region must have been open, i.e. connected to the Sun at one end. If reconnection have occurred at the heliospheric current sheet, one would expect to observe either countersteaming electrons (Gosling et al., 2006b) or a heat flux dropout (Gosling et al., 2005c) depending on whether the spacecraft traversed the reconnection site on the anti-sunward or the sunward side of the X-line. In addition, a change in the strahl direction at opposite sides of the current sheets should be observed, marking a change in the sector boundary. Moreover, the investigated event was not related to an interplanetary coronal mass ejection (ICME). Therefore, it seems that this event is likely a case of reconnection in a kinked open magnetic field line.
Figure 4 also reveals an electron conic at pitch angles of $110^{\circ}-120^{\circ}$ that continues until the trailing edge of the reconnection region. This conic started already on 22 July, 01:30 UT, i.e. too early to be related with the reconnection process. Figure $2 \mathrm{~h}$ suggests that Wind was magnetically connected to the Earth's bow shock and thus it is likely that this beam represents backstreaming electrons from the bow shock (e.g. Larson et al., 1996). During the event studied here, Wind was located relatively far away from the bow shock $\left(\sim 180 R_{E}\right)$ and although the backscattering electrons were observed down to at least $27 \mathrm{eV}$, the conic was most intense at the energies $65 \mathrm{eV}$ and above. Therefore we do not believe that the bow shock connection affects significantly 




Fig. 4. Electron pitch angle spectrograms for EESA-L energy channels $18,27,42,65,103$, and $164 \mathrm{eV}$. The data are presented in the solar wind rest frame. Two pairs of solid red lines bound the current sheet crossings while the solid black lines show the estimated locations of the separatrices. The color coding indicates the amplitude of the distribution functions in units of $\mathrm{s}^{3} \mathrm{~km}^{-3} \mathrm{~cm}^{-3}$.

our interpretation of the electron observations at energies 18 $42 \mathrm{eV}$. This electron conic at $65 \mathrm{eV}$ is enhanced when Wind enters into the reconnection region. The bow shock electrons entering the reconnection region could potentially be used to probe the magnetic field structure near the reconnection Xline but it is beyond the scope of this study.

The bidirectional electron flow at energies between 18 and $65 \mathrm{eV}$ throughout the reconnection region is visible in Fig. 4 and further highlighted in Fig. 5 that shows the electron pitch angle distributions for four low energy channels of EESA$\mathrm{L}$ (4a) before the reconnection region and (4b) inside the outflow region. In the ambient solar wind, no significant anisotropy for energies $18-42 \mathrm{eV}$ is observed; the distribution for $65 \mathrm{eV}$ electrons reflects the solar wind strahl. The approximate levels of the phase space distributions outside the reconnection region are indicated in Fig. 4 by the horizontal solid lines. It is seen from the figure that there are slight overall increases in the electron distributions between the ambient solar wind and the outflow region, but the main enhancement at phase space densities occur at pitch angles $\sim 0^{\circ}-60^{\circ}$ and at pitch angles $\sim 110^{\circ}-180^{\circ}$.

When the electron phase space densities reached maximum values during the Wind passage past the current sheets,



Fig. 5. Electron pitch angle distributions for EESA-L energy channels $18,27,42$, and $65 \mathrm{eV}$ (a) in the ambient solar wind at 01:45:00 UT, and (b) inside the outflow region at 01:46:00 UT.

the pitch angle spectrograms reveal strong enhancements broadly distributed in pitch angles at energies between 18 and $65 \mathrm{eV}$. Figure 6 shows a close-up around the current sheets at the front (left panel) and at the end (right panel) separatrix regions. The electron pitch angle spectrograms in Fig. 6 reveal a substructure within these layers. At the front side when entering the current sheet from the ambient solar wind to the outflow region, the dominant flow of thermal electrons is first along the magnetic field, but changes into a flow anti-parallel to the magnetic field during the following EESA-L sample; a close examination of the individual electron flux measurements shows that this is not an aliasing effect. Within the end separatrix region the dominant electron flow directions are reversed, i.e. the electron flow direction changes from anti-parallel to parallel when crossing the current sheet from the ambient solar wind. The observed electron flow directions are illustrated by red arrows in Fig. 1. The similar substructure within the current sheet was observed also for the EESA-L energy channels $18 \mathrm{eV}$ through $65 \mathrm{eV}$ (see Fig. 4).

\section{Discussion and summary}

In this paper we have reported high-time resolution particle observations obtained with the EESA-L and PESA-L electrostatic analyzers of the Wind satellite during the solar wind reconnection event on 22 July 1999. Solar wind reconnection is a recently found phenomena (Gosling et al., 2005a) and thus relatively little studied. The time between the observations of the separatrices at Wind was only $56 \mathrm{~s}$ and therefore the 3-s burst mode observations of EESA-L and PESA-L as well as Wind 3-s plasma measurements were essential for the identification and analysis of this event.

We estimate that Wind crossed the separatrix and the outflow regions about a thousand ion skin depths $\left(\lambda_{i}\right)$ away from 

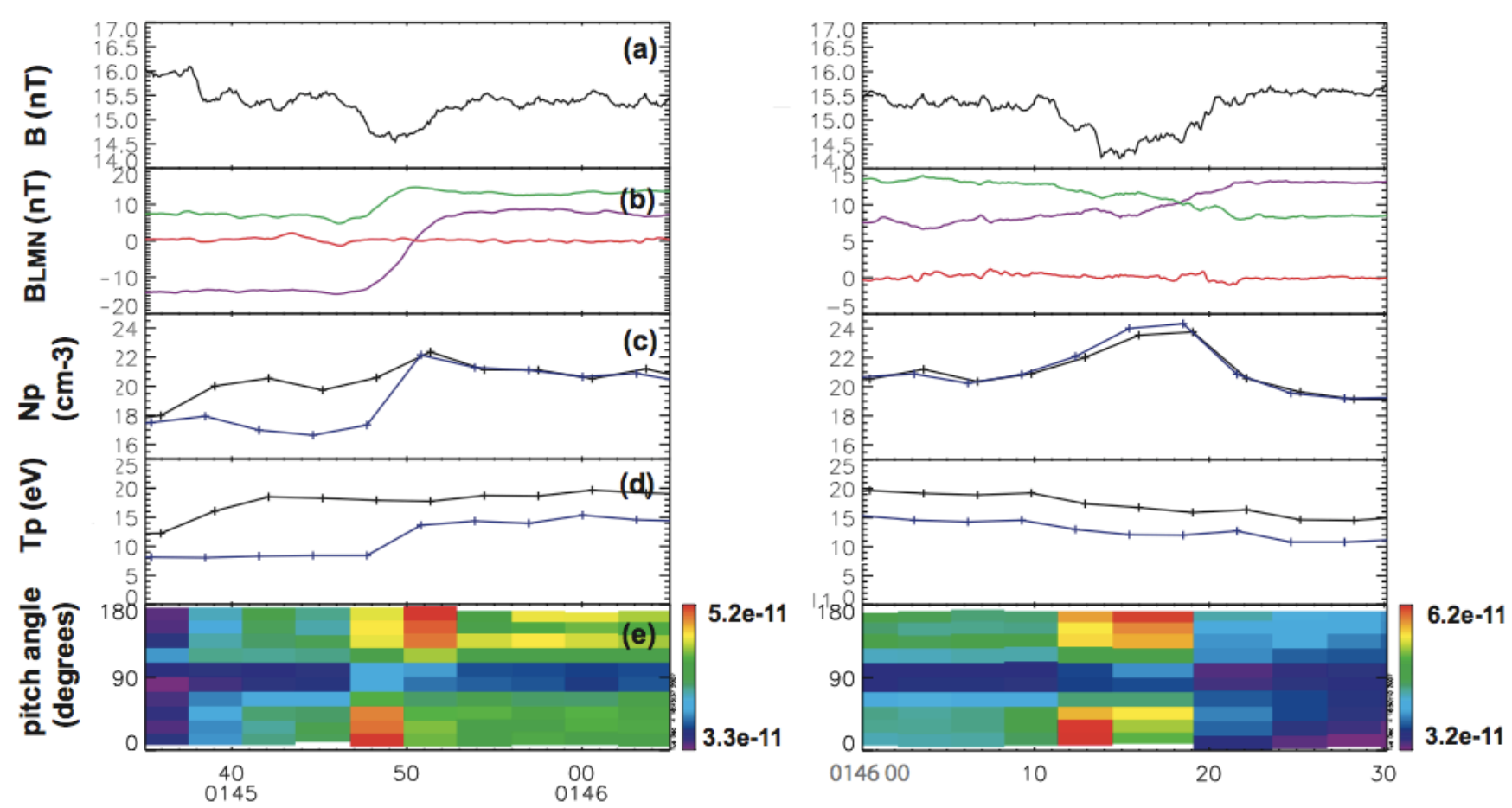

Fig. 6. Close-up around the separatrix regions at the front (left panel) and end (right panel) boundaries of the reconnection exhaust. The panels show from top to bottom: (a) the magnetic field strength, (b) the magnetic field components in the MVA coordinate system, (c) the proton density calculated on-board (blue line) and the one integrated from the 3-D distribution functions (black line), (d) the "on-board" proton temperature (blue line) and the proton temperature integrated from the 3-D distribution functions (black line), and (e) the pitch angle spectrogram of $27 \mathrm{eV}$ electrons. The color coding indicates the amplitude of the distribution functions in units of $\mathrm{s}^{3} \mathrm{~km}^{-3} \mathrm{~cm}^{-3}$.

the X-line that is significantly further than the typical crossing distances are in simulations and in most previous insitu studies. In the Earth's magnetosphere separatrix regions have been observed to maintain their internal structure several hundreds ion skin depths away from the X-line (Retinó et al., 2006; Khotyaintsev et al., 2006), but it is currently unknown how far separatrices can keep their integrity (e.g. Vaivads et al., 2006). The spatial extent of separatrix regions in the solar wind is interesting since these structures are likely to interact with the other boundaries in the solar wind and magnetosphere and the possible ramifications of the reconnection to the large-scale solar wind structure are still unknown.

The reconnection event was identified by the accelerated plasma flow confined between the bifurcated current sheet. The current sheets bounding the investigated outflow region had the width of $19 \lambda_{i}$ at the leading boundary and the width of $24 \lambda_{i}$ at the end boundary. The locations of the separatrices were estimated using an opening angle that is twice the opening angle of the outflow region. In the front separatrix region, a strong proton beam was observed. At the trailing separator boundary, the transition from outside to inside the outflow region was slow-mode like characterized by increases in the proton density and temperature and a decrease in the mag- netic field magnitude. Solar wind reconnection events, including this event, typically occur between two quite distinct plasma states (e.g. Gosling et al., 2005a, 2006a; Huttunen et al., 2007) that might account for the observed asymmetries of the separatrix regions on the opposite sides of the outflow region.

Thermal particle observations revealed several interesting features during the passage of the separatrix and outflow regions: 1) the continuation of solar wind strahl indicating that the field lines Wind crossed were open; 2) ansiotropic electron phase space distributions at energies 18 to $42 \mathrm{eV}$ with 3 ) electrons flowing towards the $\mathrm{X}$-line in the inner boundary of the outflow region and away from the X-line in the outer boundary of the outflow region and 4) a strong proton beam at the front separatrix region originating from the direction of the X-line.

In the near-Earth plasma sheet bi-directional electron pitch angle anisotropies have been reported in several studies (e.g. Hada et al., 1981; Shiokawa et al., 2003) and it has been suggested that they are produced by Fermi-type field aligned acceleration in the neutral sheet. Other mechanisms have also been suggested to produce bi-directional electrons related with reconnection events such as trapping of particles by the strong electrostatic potential (few $\mathrm{kV}$ in the magnetotail) 
across the separatrix region (Oieroset et al., 2002; Egedal et al., 2005; Wygant et al., 2005) and parallel acceleration by turbulent fields or plasma waves (e.g. Drake et al., 2003; Matsumoto et al., 2003). However, it should be noted that conditions in the magnetosphere can differ significantly from those in the solar wind and thus the comparison between the reconnection phenomena in these regions is likely not unambiguous.

Interestingly, the directions of the electron flows detected in this work near the boundaries of the outflow region were opposite to those observed in the near-Earth magnetotail (e.g. Nagai et al., 2003, see Fig. 9) and in simulations (e.g. Hoshino et al., 2001; Pritchett and Coroniti, 2004) that are consistent with the Hall current system. The event studied in this work occurred relatively far away from the X-line, the shear angle was about $114^{\circ}$ and it was associated with a relatively large guide magnetic field. However, neither large out-of-plane magnetic field or component reconnection have been shown to produce such a features (e.g. Rogers and Denton, 2003; Pritchett and Coroniti, 2004). 2-D Hall MHD simulation conducted by Scholer and Lottermoser (1998) resulted in slow mode switch-off shocks bounding the reconnection outflow region. For this case the change in the out-ofplane magnetic field component (Scholer and Lottermoser, 1998, Fig. 5) was such that the associated electron currents would be reversed compared to the usual Hall current system. However, no slow mode shocks have yet been identified in solar wind reconnection events (e.g. Gosling et al., 2005a).

Energetic ion beams have been frequently identified in the magnetotail during satellite crossings between the lobe and the plasma sheet (e.g. Takahashi and Hones, 1988; Hoshino et al., 1997). It is believed that these ions get accelerated near the X-line region. The location of the ion beam observed in this work and the lack of the ion beam at the trailing separatrix region was surprising. At the low latitude boundary layer and at the outer edge of the plasma sheet boundary layer the electron edge is observed to lie closer to the separatrix than does the ion edge due to higher parallel speeds of electrons (Takahashi and Hones, 1988; Gosling et al., 1990). However, it seems unlikely that the ion beam reported in this paper would not be related with the reconnection process since it was observed just in the separatrix region and originated from the direction of the X-line.

The event studied in this work is the first solar wind reconnection event during which sufficiently high time-resolution observations were available to probe in detail the characteristics of the separatrix and outflow domains. Thus, we do not yet know whether the above-described phenomena are common features of solar wind reconnection. More solar wind reconnection events are needed to be studied in detail to resolve these issues.

For the event studied here, we did not observe significant changes in the electron phase space densities at the highest energies of EESA-L between $103 \mathrm{eV}$ and $1.1 \mathrm{keV}$. Unfortunately, the EESA-H analyzer that measures suprathermal electrons up to $30 \mathrm{keV}$ was not operating in a burst mode. The EESA-H measurements available at a cadence of $99 \mathrm{~s} \mathrm{did}$ not show any increase in the electron distribution functions at such high energies (beyond the EESA-L energy range) during the time of the reconnection region. Similarly, Gosling et al. (2005b) reported an absence of electron flux increases during seven solar wind reconnection regions using 2-min averages of electron observations by the ACE/EPAM instrument.

The analysis conducted in this work highlights the importance of high-resolution particle measurements in studying the microphysics of reconnection. Since the 3-DP instrument on Wind is optimized to be triggered when the satellite is magnetically connected to the bow shock, there is currently a lack of high-resolution particle observations during reconnection events in the solar wind. Thus, the planning of effective criteria to trigger the burst-mode on the IMPACT particle detector on the STEREO spacecraft during passages in reconnection regions is of great importance for future investigations of small-scale reconnection processes.

Acknowledgements. We want to specially thank Matt Fillingim for his help in one of the figures of this paper. We want to acknowledge Adam Szabo for providing the high resolution Wind magnetic field data. We want to acknowledge Tai Phan and James Drake for the discussion about the electron observations. Work at the University of California was supported by NASA grant NAS5-03076.

Topical Editor I. A. Daglis thanks two anonymous referees for their help in evaluating this paper.

\section{References}

André, M., Vaivads, A., Buchert, S. C., Fazakerley, A. N., and Lahiff, A.: Thin electron-scale layers at the magnetopause, Geophys. Res. Lett., 31, L03803, doi:10.1029/2003GL018137, 2004.

Bale, S. D., Mozer, F. S., and Phan, T.: Observation of lower hybrid drift instability in the diffusion region at a reconnecting magnetopause, Geophys. Res. Lett., 29(24), 2180, doi:10.1029/2002GLL016113, 2002.

Cattell, C., Dombeck, J., Wygant, J., Drake, J. F., Swisdak, M., Keith, W., Fazakerley, A., André, M., Lucek, E., and Balogh, A.: Cluster observations of electron holes in association with magnetotail reconnection and comparison to simulations, J. Geophys. Res., 110, A01211, doi:10.1029/2004JA010519, 2005.

Davis, M. S., Phan, T. D., Gosling, J. T., and Skoug, R. M.: Detection of oppositely directed reconnection jets in a solar wind current sheet, Geophys. Res. Lett., 33, L19102, doi:10.1029/2006GL026735, 2006.

Drake, J. F., Swisdak, M., Cattell, C., Shay, M. A., Rogers, B. N., and Zelier, A.: Formation of electron holes and particle energization during magnetic reconnection, Science, 299, 873-877, 2003.

Egedal, J., Oieroset, M., Fox, W., and Lin, R. P.: In situ discovery of an electrostatic potential, trapping electrons and mediating fast reconnection in the Earth's magnetotail, Phys. Rev. Lett., 94, 025006, doi:10.1103/PhysReVLett.94.025006, 2005. 
Filbert, P. C. and Kellogg, P.: Electrostatic noise at the plasma frequency beyond the Earth's bow shock, J. Geophys. Res., 84(A4), 1369-1381, 1979.

Gosling, J. T., Thomsen, M. F., Bame, S. J., Onsagner, T. G., and Russell, C. T.: The electron edge of the low latitude boundary layer during accelerated flow events, Geophys. Res. Lett., 17, 1833-1836, 1990.

Gosling, J. T., Skoug, R. M., McComas, D. J., and Smith, C. W.: Direct evidence for magnetic reconnection in the solar wind near 1 AU, J. Geophys. Res., 110, A01107, doi:10.1029/2004JA010809, 2005a.

Gosling, J. T., Skoug, R. M., Haggerty, D. K., and McComas, D. J.: Absence of energetic particle effects associated with magnetic reconnection exhausts in the solar wind, Geophys. Res. Lett., 32, L14113, doi:10.1029/2055GL023357, 2005b.

Gosling, J. T., Skoug, R. M., McComas, D. J., and Smith, C. W.: Magnetic disconnection from the Sun: Observations of a reconnection exhaust in the solar wind at the heliospheric current sheet, Geophys. Res. Lett., 32, L05105, doi:10.1029GL022406, 2005c.

Gosling, J. T., Eriksson, S., Skoug, R. M., McComas, D. J., and Forsyth R. J.: Petschek-type reconnection exhausts in the solar wind well beyond 1 AU, The Astrophys. J., 644, 613-621, 2006 a.

Goslin, J. T., McComas, D. J., Skoug, R. M., and Smith, C. W.: Magnetic reconnection at the heliospheric current sheet and the formation of closed magnetic field lines in the solar wind, Geophys. Res. Lett., 33, L17102, doi:10.1029/2006GL027188, 2006b.

Gosling, J. T., Eriksson, S., Phan, T. D., Larson, D. E., Skoug, R. M., and McComas, D. J.: Direct evidence for prolonged magnetic reconnection at a continuous $\mathrm{x}$-line within the heliospheric current sheet, Geophys. Res. Lett., 34, L06102, doi:10.1029/2006GL029033, 2007.

Hada, T., Nishida, A., Terasawa, T., and Hones, Jr., E. W.: Bidirectional electron pitch angle anisotropy in the plasma sheet, J.Geophys. Res., 86, 11 211-11 224, 1988.

Hoshino, M., Nishida, A. Mukai, T., Kokubun, S., and Yamamoto, T.: Ion dynamics of magnetic reconnection in the magnetotail, Adv. Space. Res., 19, 1827-1853, 1997.

Hoshino, M., Hiraide, K., and Mukai, T.: Strong electron heating and non-Maxwellian behaviour in magnetic reconnection, Earth Planets Space, 53, 627-634, 2001.

Huttunen, K. E. J., Bale, S. D., Phan, T. D., Davis, M., and Gosling, J. T.: WIND/WAVES observations of high frequency plasma waves in solar wind reconnection exhausts, J. Geophys. Res., 111, A10102, doi:10.1029/2006JA011863, 2007.

Khotyaintsev, Yu., Vaivads, V., Retinó, A., André, A., Owen, C. J., and Nilsson, H.: Formation of inner structure of a reconnection separatrix region, Phys. Rev. Lett., 97, 205003 , doi:10.1103/PhysRevLett.97.205003, 2006.

Larson, D. E., Lin, R. P., McFadden, J., Ergun, R. E., Carlson, C. W., Anderson, K. A., Phan, T. D., McCarthy, M., Parks, G. K., Rme, H., Bosqued, J. M., d'Uston, C., Wenzel, K.-P., Sanderson, T. R., and Lepping, R. P.: Probing the Earth's bow shock with upstream electrons, Geophys. Res. Lett., 23, 2203-2206, 1996.

Lin, R. P, Anderson, K. A., Ashford, S., Carlson, C., Curtis, D., Ergun, R., Larson, D., McFadden, J., McCarthy, M., Parks, G. K., Réme, H., Bosqued, J. M., Coutelier, J., Cotin, F., d'Uston, C., Wenzel, K.-P., Sanderson, T. R., Henrion, J., Ronnet, J. C., and
Paschmann, G.: A three-dimensional (3-D) plasma and energetic particle experiment for the WIND spacecraft of the ISTP/GGS mission, Space Sci. Rev., 71, 125-153, 1995.

Matsumoto, H., Deng, X. H., Kojima, H., and Anderson, R. R.: Observation of electrostatic solitary waves associated with reconnection on the dayside magnetopause boundary, Geophys. Res. Lett., 30(6), 1326, doi:10.1029/2002GL016319, 2003.

Mozer, F. S.: Criteria for and statistics of electron diffusion regions associated with sub-solar magnetic field reconnection, J. Geophys. Res., 110, A12222, doi:10.1029/2005JA011258, 2005.

Nagai T., Shinohara, I., Fujimoto, M., Machida, S., Nakamura, R., Saito, Y., and Mukai, T.: Structure of the Hall current system in the vicinity of the magnetic reconnection site, J. Geophys. Res., 108(A10), 1357, doi:10.1029/2003JA009900, 2003.

Oieroset, M., Lin, R. P., Phan, T. D., Larson, D. E., and Bale, S. D.: Evidence for electron acceleration up to $\sim 300 \mathrm{KEV}$ in the magnetic reconnection diffusion region of Earth's magnetotail, Phys. Rev. Lett., 89, 195001, 2002.

Petschek, H. E.: Magnetic Field Annihilation, NASA Spec. Publ. SP-50, 425, 1964.

Phan, T. D., Gosling, J. T., Davis, M. S., Skoug, R. M., Oieroset, M., Lin, R. P., Lepping, R., McComas, D. J., Smith, C. W., Reme, H. M., and Balogh, A.: In-situ detection of a magnetic reconnection region extending more than 390 Earth radii in the solar wind, Nature, 439, 175-178, 2006.

Pritchett, P. L. and Coroniti, F. V.: Three dimensional collisionless magnetic reconnection in the presence of a guide field, J. Geophys. Res., 109, A01220, doi:10.1029/2003JA009999, 2004.

Retinó, A., Vaivads, A., André, M., Saharoui, F., Khotyaintsev, Pickett, J. S., Bavassano Cattaneo, M. S., Marcucci, M. F., Morooka, M., Owen, C. J., Buchert, S. C., and Cornilleau-Wehrlin, N.: The structure of the separatrix region close to a magnetic reconnection X-line: Cluster observations, Geophys. Res. Lett., 33, L06101, doi:10.1029/2005GL024650, 2006.

Rogers, B. N. and Denton, R. E.: Signatures of collisionless magnetic reconnection, J. Geophys. Res., 108(A3), 1111, doi:10.1029/2002JA009699, 2003.

Salem, C., Bosqued, J.-M., Larson, D. E., Mangeney, A., Maksimovic, M., Perche, C., Lin, R. P., and Bougeret, J.-L.: Determination of accurate solar wind electron parameters using particle detectors and radio wave receivers, J. Geophys. Res., 106, 21 701-21 717, 2001.

Scholer, M. and Lottermoser, R.-F.: on the kinetic structure of the magnetotail reconnection layer, Geophys. Res. Lett., 25, 23813284, 1998.

Shiokawa, K., Baumjohann, W., and Paschmann, G.: Bi-directional electrons in the near-Earth plasma sheet, Ann. Geophys., 21, 1497-1507, 2003, http://www.ann-geophys.net/21/1497/2003/.

Slavin, J. A. and Holzer, R. E.: Solar wind flow about the terrestrial planets: 1 . Modeling bow shock position and shape, J. Geophys. Res., 86, 11 401-11418, 1981.

Sonnerup, B. U. Ö. and Cahill Jr., L. J.: Magnetopause structure and attitude from Explorer 12 observations, J. Geophys. Res., 96(72), 171-183, 1967.

Takahashi, K. and Hones Jr., E. W.: ISEE 1 and 2 observations of ion distributions at the plasma sheet-tail lobe boundary, J. Geophys. Res., 93, 8558-8582, 1988.

Vaivads, A., Retinó, A., and André, M.: Microphysics of magnetic reconnection, Space Sci. Rev., 122, 19-27, 2006. 
Wygant, J. R., Cattell, C. A., Lysak, R., Song, Y., Dombeck, J., McFadden, J., Mozer, F. S., Carlson, C. W., Parks, G., Lucek, E. A., Balogh, A., Andre, M., Reme, H., Hesse, M., and Mouikis, C.: Cluster observations of an intense normal component of the electric field at a thin reconnecting current sheet in the tail and its role in the shock-like acceleration of the ion fluid into the separatrix region, J. Geophys. Res., 110, A09206, doi:10.1029/2004JA010708, 2005. 